\title{
LOS CONOS PIROCLÁSTICOS DE SABANA REDONDA: COMPONENTE MAGMATICO ENRIQUECIDO DEL VOLCÁN POÁS, COSTA RICA
}

\author{
Esteban Gazel ${ }^{1 *} \&$ Pablo Ruiz ${ }^{2}$ \\ ${ }^{1}$ Department of Geological Sciences, Rutgers University, \\ 610 Taylor Rd. Piscataway, NJ 08854 USA. \\ ${ }^{2}$ Escuela Centroamericana de Geología, Universidad de Costa Rica, \\ Apartado 214-2060 UCR \\ *Autor para contacto: egazel@eden.rutgers.edu
}

(Recibido 10/10/06; aceptado 03/11/06)

\begin{abstract}
We describe the stratigraphy and geochemistry of tephras and lavas related with 5 cinder cones in a north-south alignment on the southern flank of Poás volcano. The local geologic basement is composed mainly by lava flows, with the northwest dominated by the Achiote basaltic-andesites and the rest of the area by Poasito andesites, this last cogenetic with the cinder cones. All of these units are generally covered by a thin layer of the upper section of the Poás Lapilli Tuff. Field evidence suggests that the cinder cones were not contemporaneous but represent different magmatic pulses. The magmas that produced these cinder cones represent the most enriched magmatic component of Poás volcano. Based on the available geochemical data, we define two magmatic components for Poás: 1) The Sabana Redonda component $\left(\mathrm{TiO}_{2}>1 \%\right.$, relatively enriched in HFSE and other incompatible elements), which is also present in the Paleo-Poás, Poás lapilli tuff y and some Botos crater lavas. 2) The von Frantzius component $\left(\mathrm{TiO}_{2}<0.8 \%\right)$, which is present at lavas from the main crater, von Frantzius cone, Hule maar, and in some Botos crater lavas. Intermediate lavas $\left(\mathrm{TiO}_{2} 0.8\right.$ $1 \%)$ are hybrids between these two components. Both components share the same OIB-like source, although the Sabana Redonda component requires a relatively lower degree of partial melting, produced primarily by a decompression mechanism, which may be related with the extension generated within the Poás volcano-tectonic fracture. The von Frantzius component represents magmas produced primarily by flux melting related with the subduction processes.
\end{abstract}

Key words: pyroclastic cones, volcanology, geochemistry, tephro-stratigraphy

RESUMEN: En el flanco sur del volcán Poás se encontraron los relictos de 5 conos piroclásticos alineados en una dirección norte-sur. El basamento local está representado por coladas de lava, en el sector noroeste por las andesitas basálticas Achiote y en la resto del área de estudio por las andesitas Poasito. Estas últimas son cogenéticas con los conos piroclásticos. Todas las unidades anteriores se encuentran cubiertas por la sección superior de la Toba de Lapilli Poás. Las evidencias de campo sugieren que la actividad ígnea relacionada con los conos piroclásticos no es contemporánea, sino más bien representa diferentes pulsos de actividad. Los magmas 


\begin{abstract}
que originaron los conos piroclásticos representan el extremo enriquecido de los componentes del volcán Poás. Con base en los datos geoquímicos disponibles se definieron dos componentes para los magmas del Poás: 1) Componente Sabana Redonda $\left(\mathrm{TiO}_{2}>1 \%\right.$, enriquecido en HFSE y otros elementos incompatibles), presente además en las lavas Paleo-Poás, la Toba de Lapilli Poás y algunas lavas del cráter Botos. 2) Componente von Frantzius $\left(\mathrm{TiO}_{2}<0,8 \%\right)$, presente en las lavas de la zona del cráter Principal, el cono von Frantzius, la laguna de Hule y algunas lavas del cráter Botos. Las lavas con composiciones intermedias $\left(\mathrm{TiO}_{2}\right.$ 0,8-1\%) son híbridos entre estos dos componentes. Ambos componentes comparten una misma fuente tipo OIB, sin embargo, el componente Sabana Redonda requiere un menor grado de fusión parcial por medio de decomprensión, posiblemente relacionado con la extensión dentro de la fractura volcanotectónica del Poás, mientras que el componente von Frantzius representa los magmas originados por medio de fusión por fluidos de subducción.

Palabras clave: conos piroclásticos, vulcanología, geoquímica, tefroestratigrafía.
\end{abstract}

\section{INTRODUCCIÓN}

La cordillera volcánica Central de Costa Rica corresponde con una cadena montañosa de $80 \mathrm{~km}$ de longitud y una altura máxima de 3432 m. Está conformada por cinco macizos volcánicos, que del noroeste al sureste son: Platanar - Porvenir, Poás, Barva, Irazú y Turrialba. La actividad volcánica es el producto de la subducción de la placa del Coco bajo la placa Caribe, la cual ocurre a una velocidad aproximada de $10 \mathrm{~mm} / \mathrm{año} \mathrm{(DeMets,} \mathrm{2001).}$ Barquero \& Malavassi (1982) reportaron la existencia de al menos 4 conos piroclásticos, entre las poblaciones de Sabana Redonda y Bajos del Tigre, en el sector sur del volcán Poás, los cuales denominaron conos Sabana Redonda. Estos conos se encuentran constituidos por secuencias ligeramente estratificadas de cenizas, lapilli, bombas y aglomerados (Alvarado, 2000). Prosser (1983) sugiere que existen al menos dos conos contemporáneos en la zona de Sabana Redonda y otro en los alrededores de Bajos del Tigre. Tournon (1984) menciona que el material de estos conos es el producto de erupciones estrombolianas y relaciona genéticamente las coladas afíricas en los alrededores de la zona con los conos piroclásticos.

Los análisis químicos realizados por Prosser \& Carr (1987) muestran que las lavas en los alrededores de Sabana Redonda y los componentes juveniles de los conos piroclásticos varían de basaltos a andesitas altas en $\mathrm{TiO}_{2}(>1$ $\%)$. Los análisis químicos de la Toba de Lapilli Poás muestran que estos depósitos presentan un carácter andesítico basáltico y un menor enriquecimiento en $\mathrm{TiO}_{2}$.

El área de estudio se localiza en los alrededores de los conos piroclásticos de Sabana Redonda (Fig. 1). El objetivo principal de este trabajo es mostrar las relaciones estratigráficas y geoquímicas de las unidades geológicas cartografiadas y la relación de estas unidades con los componentes magmáticos del volcán Poás, que se definen en este trabajo.

\section{METODOLOGÍA}

Se realizó un cartografiado geológico general como parte del curso de Campaña Geológica de la Escuela Centroamericana de Geología de la Universidad de Costa Rica, el cual posteriormente se detalló y modificó. Además, se revisaron los archivos de los pozos para la extracción de agua del SENARA y se hicieron estudios fotogeológicos de la zona. Posteriormente se hicieron múltiples visitas al campo para detallar las secciones medidas y realizar muestreos geoquímicos de las diferentes unidades geológicas. Se hizo un mapa de isopletas de tamaños máximos ( $\varphi$ máx.) de los componentes juveniles de la sección superior de la Toba de Lapilli Poás, con el propósito de mostrar la distribución regional de esta unidad. Estas isopletas se construyeron con una base de datos que se encuentra a solicitud de los autores, que incluye datos de $\varphi$ máx, de Prosser (1983), Montes (com. pers., 2006) y datos no publicados de los autores. Para diferenciar entre los depósitos de los conos piroclásticos de Sabana Redonda y la Toba de Lapilli Poás se muestrearon las secciones menos alteradas y se realizaron 7 análisis químicos de elementos mayores (\% del peso total). De las muestras seleccionadas, se trituraron aproximadamente $100 \mathrm{~g}$ de los componentes juveniles y posteriormente se secaron $26 \mathrm{~g}$ del material en un molde de aluminio a una temperatura de $90^{\circ} \mathrm{C}$ por 24 horas. De este material se pulverizaron $12 \mathrm{~g}$ en 
un molino de cilindros. Con el material pulverizado se construyó una pastilla a una presión de 15 t/ $\mathrm{cm}^{2}$. Los análisis se realizaron mediante la técnica de fluorescencia de rayos X, con el equipo FISONS ARL 8660 S, en el laboratorio de CEMEX Costa Rica, estandarizado y certificado en 1999 por Construction Technology Laboratories Engineers and Construction Technology Consultants con sede en Illinois, Estados Unidos. Además se recopilaron análisis químicos de elementos mayores (\% del peso total) y trazas (ppm) del volcán Poás de la base de datos CAGeochem (Prosser \& Carr, 1987; Patino et al., 2000; Carr, 2002), los cuales se graficaron y modelaron usando el programa IGPET 2005. Tanto la base de datos recopilada como los nuevos análisis de elementos mayores se encuentran disponibles a solicitud de los autores.

\section{ESTRATIGRAFÍA VOLCÁNICA}

\section{Andesitas basálticas Achiote}

Se encuentran expuestas en las quebradas y ríos al oeste de la zona de estudio. El mejor afloramiento se localiza en el río Achiote (510,18 1237,47 ; Fig. 1) donde aflora un frente de colada de lava masiva con fracturas de enfriamiento y un espesor máximo de $15 \mathrm{~m}$.

Generalmente son de color gris claro y presentan una textura afanítica-porfirítica con fenocristales de plagioclasa centimétricos y algunos piroxenos milimétricos. Presenta colores con tonalidades moradas y verdosas en las pátinas de meteorización. Petrográficamente presenta una textura hipocristalina-porfirítica con fenocristales de plagioclasas (15-20\%) idiomórficas a hipidiomórficas con un largo máximo de $1 \mathrm{~cm}$, clinopiroxenos (9\%) hipidiomórficos con un largo máximo de 1,5 $\mathrm{mm}$, ortopiroxenos (2\%) hipidiomórficos con un largo máximo de $3 \mathrm{~mm}$ y olivinos (1-3\%) idiomórficos a hipidiomórficos con un largo máximo de $0,3 \mathrm{~mm}$ en una matriz hialopilítica.

\section{Andesitas Poasito}

Representa la unidad de mayor extensión y espesor en el área de estudio. Las mejores exposiciones se encuentran en el río Poasito, en el cauce alto del río Poás y en las quebradas en los alrededores de Bajos del Tigre y Sabana Redonda (Fig. 1).

Agrupa coladas andesíticas basálticas a andesíticas con una estructura masiva y textura fluidal (pliegues, fallas inversas y estructuras de cizalle interno, remanentes del movimiento de la colada). Además, se caracterizan por presentar columnas de enfriamiento poco desarrolladas y en los cortes transversales, en el río Poasito se aprecian las autobrechas superiores y basales.

Macroscópicamante el color varía de gris claro a oscuro (con el aumento del contenido de vidrio) en las superficies sanas y tonos naranja en las pátinas de meteorización. Estas lavas tienen una textura afanítica-fluidal con pocos cristales milimétricos de plagioclasa y piroxenos en una matriz vítrea. Microscópicamente, se caracterizan por presentar una textura hipocristalina-porfirítica, con un matiz pilotaxítica y un contenido de fenocristales que no supera el 5\%. Dentro de estos fenocristales se encuentran plagioclasas (2\%) hipidiomórficas con un largo máximo de 0,6 mm; clinopiroxenos (1\%) hipidimórficos con un largo máximo de $0,5 \mathrm{~mm}$ y ortopiroxenos (1\%) hipidiomórficos con un largo máximo de $0,4 \mathrm{~mm}$.

Esta unidad presenta un espesor observado de $20 \mathrm{~m}$ en el río Poasito, no obstante, con la información recopilada de perforaciones de la zona en la base de datos del SENARA, el espesor de esta unidad podría superar los 80 metros. En el río Poasito y al sur de la población de Sabana Redonda, se observan al menos 2 coladas de lava intercaladas entre los depósitos piroclásticos de la Toba de Lapilli Poás. En ambas localidades se observó un contacto quemado de color rojizo de unos $20 \mathrm{~cm}$. No se observó la presencia de paleosuelos orgánicos, por lo que se puede inferir que los eventos piroclásticos y efusivos fueron relativamente contemporáneos. Con base en el estudio de las fotografías aéreas se sugiere la existencia de al menos 4 coladas, con una fuente cercana a la fractura volcanotectónica del Poás (Fig. 1). Las características petrográficas y reológicas, y la localización de la fuente de estas coladas dentro de la fractura volcanotectónica del Poás, sugieren que se originaron por medio de un volcanismo fisural. 



Fig. 1. Mapa geológico de los alrededores de los conos piroclásticos de Sabana Redonda. F.V.P., fractura volcanotectonica del Poás. 
Cuadro 1

Composición petrográfica de los bloques (inclusiones) dentro de los conos piroclásticos

\begin{tabular}{|c|c|c|c|c|}
\hline Código & Coordenadas & Descripción Microscópica & Clasificación & Correlación \\
\hline SR-27704-9a & $512,52 / 235,50$ & $\begin{array}{l}\text { Textura hipocristalina-porfirítica, plagioclasa } \\
(2 \%) \text {, clinopiroxeno }(<1 \%) \text {, magnetita }(1 \%) \\
\text { en una matriz hialina de aspecto fluidal. }\end{array}$ & $\begin{array}{l}\text { andesita } \\
\text { basáltica }\end{array}$ & $\begin{array}{l}\text { andesitas } \\
\text { Poasito }\end{array}$ \\
\hline SR-27704-9b & $512,52 / 235,50$ & $\begin{array}{l}\text { Textura fanerítica-porfirítica, plagioclasa } \\
(15 \%) \text {, cumulitos de olivino }(2 \%) \text {, magnetita } \\
(3 \%) \text {, clinopiroxeno }(2 \%) \text { en una matriz } \\
\text { hialopilitica. }\end{array}$ & $\begin{array}{l}\text { andesita } \\
\text { basáltica }\end{array}$ & $\begin{array}{l}\text { andesitas } \\
\text { basálticas } \\
\text { Achiote }\end{array}$ \\
\hline SR-27704-10 & $512,28 / 235,04$ & $\begin{array}{l}\text { Textura fanerítica-porfirítica, plagioclasa } \\
(20 \%) \text {, cumulitos de olivino ( } 4 \%) \text {, magnetita } \\
(3 \%) \text {, clinopiroxeno }(1 \%) \text { en una matriz } \\
\text { hialopilítica. }\end{array}$ & $\begin{array}{l}\text { andesita } \\
\text { basáltica }\end{array}$ & $\begin{array}{l}\text { andesitas } \\
\text { basálticas } \\
\text { Achiote }\end{array}$ \\
\hline SR-27704-12 & $512,28 / 235,04$ & $\begin{array}{l}\text { Textura fanerítica-porfiritica, } \\
\text { megafenocristales plagioclasa }(15 \%) \text {, olivino } \\
(5 \%) \text {, clinopiroxeno }(8 \%) \text {, magnetita }(1 \%) \\
\text { en una matriz hialopilítica. }\end{array}$ & $\begin{array}{l}\text { basalto con } \\
\text { olivino }\end{array}$ & $\begin{array}{l}\text { Paleo- } \\
\text { Poás? }\end{array}$ \\
\hline P-033-01 & $512,61 / 23,37$ & $\begin{array}{l}\text { Textura fanerítica-porfirítica, plagioclasa } \\
(18 \%) \text {, cumulitos de olivino }(2 \%) \text {, magnetita } \\
(3 \%) \text {, clinopiroxeno }(1 \%) \text { en una matriz } \\
\text { hialopilítica. }\end{array}$ & $\begin{array}{l}\text { andesita } \\
\text { basáltica }\end{array}$ & $\begin{array}{l}\text { andesitas } \\
\text { basálticas } \\
\text { Achiote }\end{array}$ \\
\hline P-033-02 & $512,61 / 23,37$ & $\begin{array}{l}\text { Textura hipocristalina-porfirítica, plagioclasa } \\
(2 \%) \text {, clinopiroxeno }(2 \%) \text {, olivino }(<1 \%) \\
\text { magnetita }(1 \%) \text { en una matriz hialina de } \\
\text { aspecto fluidal. }\end{array}$ & $\begin{array}{l}\text { andesita } \\
\text { basáltica }\end{array}$ & $\begin{array}{l}\text { andesitas } \\
\text { Poasito }\end{array}$ \\
\hline
\end{tabular}

\section{Conos piroclásticos Sabana Redonda}

Las mejores exposiciones se encuentran en los tajos Rocha, Cahrmi, Poás, Poasito y Los Mora. Afloramientos menores se encuentran en canteras secundarias en los viveros de Bajos del Tigre y en los cortes de carretera entre las poblaciones de Sabana Redonda y Poasito (Fig. 1).

Esta unidad está compuesta por depósitos de bombas y bloques en una matriz de lapilli (Fig. 2), niveles de aglomerados y pequeñas coladas de lava aglutinada en la base (Fig. 3). En algunos cortes se pueden apreciar estructuras que se interpretan como conductos volcánicos o posibles chimeneas de desgasificación (Fig. 4). La fuente de emisión de esta unidad corresponde con 5 conos piroclásticos alineados en una dirección norte-sur, desde la población de Sabana Redonda hasta el hito Carlos (Fig. 1). Los espesores alcanzan hasta $60 \mathrm{~m}$ en las localidades cercanas a los puntos de emisión. En los cortes de carretera y caminos secundarios el espesor observado difícilmente supera los $5 \mathrm{~m}$.
Los depósitos presentan una estratificación poco evidente y una selección de mala a moderada (Fig. 2), la cual mejora al alejarse de la fuente. Los fragmentos juveniles corresponden principalmente con bombas escoriáceas tipo fusiforme, corteza de pan e irregulares de hasta $2 \mathrm{~m}$ de largo. Menos frecuentes son bloques angulares de lavas de las andesitas Poasito, de las andesitas basálticas Achiote y otros difícilmente correlacionables con las unidades definidas en este trabajo. El cuadro 1 resume las características petrográficas de estos bloques y muestra una posible correlación de ellos.

Los componentes juveniles presentan un color naranja oscuro, producto de una oxidación del hierro a alta temperatura, al entrar en contacto con el aire. Sin embargo, dentro de los conos piroclásticos también se observan niveles subordinados de un color gris claro, que sugiere condiciones ambientales relativamente secas que no favorecieron la oxidación del material (Fisher \& Schminke, 1984). Las bombas presentan un 30-35\% de vesículas, en 
una matriz vítrea con una textura fluidal similar a las lavas de las andesitas Poasito.

Las coladas de lava (Fig. 3) presentan un color gris claro y una textura afírica-fluidal muy similar a las andesitas Poasito y no superan los 4 $\mathrm{m}$ de espesor. Estas lavas se encuentran intercaladas en los niveles basales de los conos piroclásticos en los tajos Cahrmi y Rocha y probablemente representen coladas de aglutinados que se soldaron a altas temperaturas.

Una datación radiométrica con C-14 en material carbonizado por los depósitos de los conos de los alrededores de la localidad de Sabana Redonda dio una edad de 33 ka (G.E. Alvarado, com. pers., 2005).

\section{Toba de Lapilli Poás}

Esta unidad fue definida por Prosser (1983). Agrupa las diferentes tobas de lapilli del sector cuspidal del volcán Poás. Las mejores exposiciones dentro del área de estudio se aprecian en los cortes de carretera entre las poblaciones de Sabana Redonda y Poasito, donde alcanzan hasta $5 \mathrm{~m}$ de espesor. Muestran un color gris claro cuando se encuentran frescos y colores que varían de café a naranja cuando se encuentran meteorizados. Generalmente estas tobas cubren las otras



Fig. 2. Depósitos de bombas y bloques de uno de los conos piroclásticos en un corte en el interior de los viveros de Bajos del Tigre $(513,30 / 243,46)$.

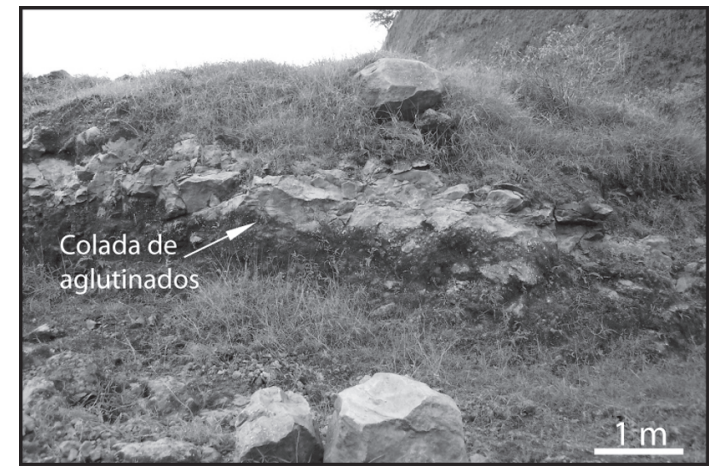

Fig. 3: Colada de aglutinados, base del tajo Rocha $(512,83 / 233,16)$.

unidades del área de estudio, sin embargo, no se muestra en el mapa geológico con el objetivo de detallar las unidades lávicas y los conos piroclásticos. La figura 5 muestra las isopletas construidas con el tamaño máximo de los componentes juveniles de la capa superior de esta unidad.

Los depósitos corresponden con tobas de lapilli fino a grueso, escoriáceo con líticos de lavas angulares, que evidencian una fragmentación en seco (vesículas dentro de los materiales juveniles) aunque también se aprecian niveles intercalados de tobas de ceniza de menor espesor (Fig. 6). De acuerdo con el grado y tipo de fragmentación, se interpreta que se originó a partir de erupciones de tipo estrombolianas a vulcanianas (Fisher \& Schminke, 1984).

Dentro de esta unidad se encuentran intercalados los depósitos de los conos piroclásticos Sabana Redonda (Fig. 6) y las andesitas Poasito. La sección inferior de esta toba se puede distinguir por presentar dos juegos seguidos de paquetes no mayores a $20 \mathrm{~cm}$ de ceniza-lapilli medio, la cual generalmente se encuentra sobreyacida por las coladas de andesitas Poasito o los depósitos de los conos piroclásticos Sabana Redonda (Figs. 6 y 7). La sección superior representa los últimos eventos volcánicos preservados en el área de estudio, caracterizados por dos niveles centimétricos de lapilli grueso escoriáceo con cristales milimétricos de clinopiroxenos, fácilmente reconocibles en la mayoría de los afloramientos. Estos niveles se encuentran intercalados con capas centimétricas a métricas de lapilli fino a medio y tobas de ceniza subordinadas (Figs. 6 y 8 ). 




Fig. 4. Conducto volcánico/chimenea de desgasificación en un corte de explotación, en uno de los conos piroclásticos de los alrededores de la localidad de Sabana Redonda (512,73/232,95).

Prosser \& Carr (1987) realizaron una datación radiométrica C-14 de la Toba de Lapilli Poás, que muestra una edad superior a los $40 \mathrm{ka}$.

\section{GEOLOGÍA ESTRUCTURAL}

\section{Fractura volcanotectónica del Poás}

La parte central del área de estudio se encuentra dentro de una fractura volcanotectónica de rumbo norte-sur que comprende el alineamiento de los conos piroclásticos Sabana Redonda, el edifico actual de volcán Poás (cráter principal, cráter Botos y el cono von Frantzius), el volcán Congo y los cráteres de explosión (maar) de Hule y río Cuarto (Prosser, 1986; Alvarado, 2000; Denyer et al. 2003).

Los conos piroclásticos Sabana Redonda se encuentran en los bordes de una depresión nortesur dentro de la cual se encuentran la localidad de Bajos del Tigre (Fig.1). Esta depresión está bordeada al este y al oeste por escarpes lineales de pendientes fuertes $\left(25-45^{\circ}\right)$ de hasta $70 \mathrm{~m}$ de altura. No se encuentran evidencias de campo de los planos principales de esta fractura, aunque se observaron varias manifestaciones menores de fallamiento. En el escarpe este se encontró una zona de falla con una separación vertical de $25 \mathrm{~cm}$ (512,5/231, Fig. 1), la cual corta unos depósitos piroclásticos alterados que subyacen a la Toba de Lapilli Poás y cuyo plano principal presenta una inclinación $270 / 60^{\circ}$ (Fig. 9). Otra zona de falla, en el escarpe este $(512,8 / 234,9)$ corta las andesitas Poasito, presenta un plano $260 / 75^{\circ}$ con estrías que se inclinan $85^{\circ}$ al noroeste y presenta un movimiento relativo normal. En el escarpe oeste se encontró una zona de falla que corta los depósitos de la Toba de Lapilli Poás con un plano principal 90/70 y una separación aparente normal.

Al este de la depresión principal se encontró un sistema de fallas $(513,5 / 233,2)$ que cortan la sección inferior de la Toba de Lapilli Poás y los conos piroclásticos Sabana Redonda, con al menos 3 planos de falla que se inclinan $90 / 60^{\circ}$, $112 / 60^{\circ}, 110 / 65^{\circ}$, de este a oeste respectivamente (Fig. 7). Las separaciones aparentes son inversas o normales y no superan $\operatorname{los} 2 \mathrm{~cm}$.

\section{Falla Río Poás}

Se ubica en las laderas y el cauce del río Poás (Fig. 1). Presenta un rumbo noreste-suroeste y un movimiento relativo sinestral con base en las evidencias fotogeológicas, que incluyen los desplazamientos sinestrales del escarpe de la falla de Alajuela y del frente montañoso al sur de la población de Sabana Redonda.

Durante el trabajo de campo se observaron dos zonas de falla en el río Poás posiblemente relacionadas con esta estructura. La primera $(514,8 / 234,6)$ comprende un área cizallada que corta las andesitas Poasito, con planos estriados y generación de arcillas de falla. Presentan una inclinación $260 / 85^{\circ}$ y un movimiento relativo sinestral. La segunda zona de falla $(513,5 / 232,0)$ corta los depósitos de la Toba de Lapilli Poás y presenta un plano que se inclina $270 / 65^{\circ}$ con un movimiento relativo sinestral. 


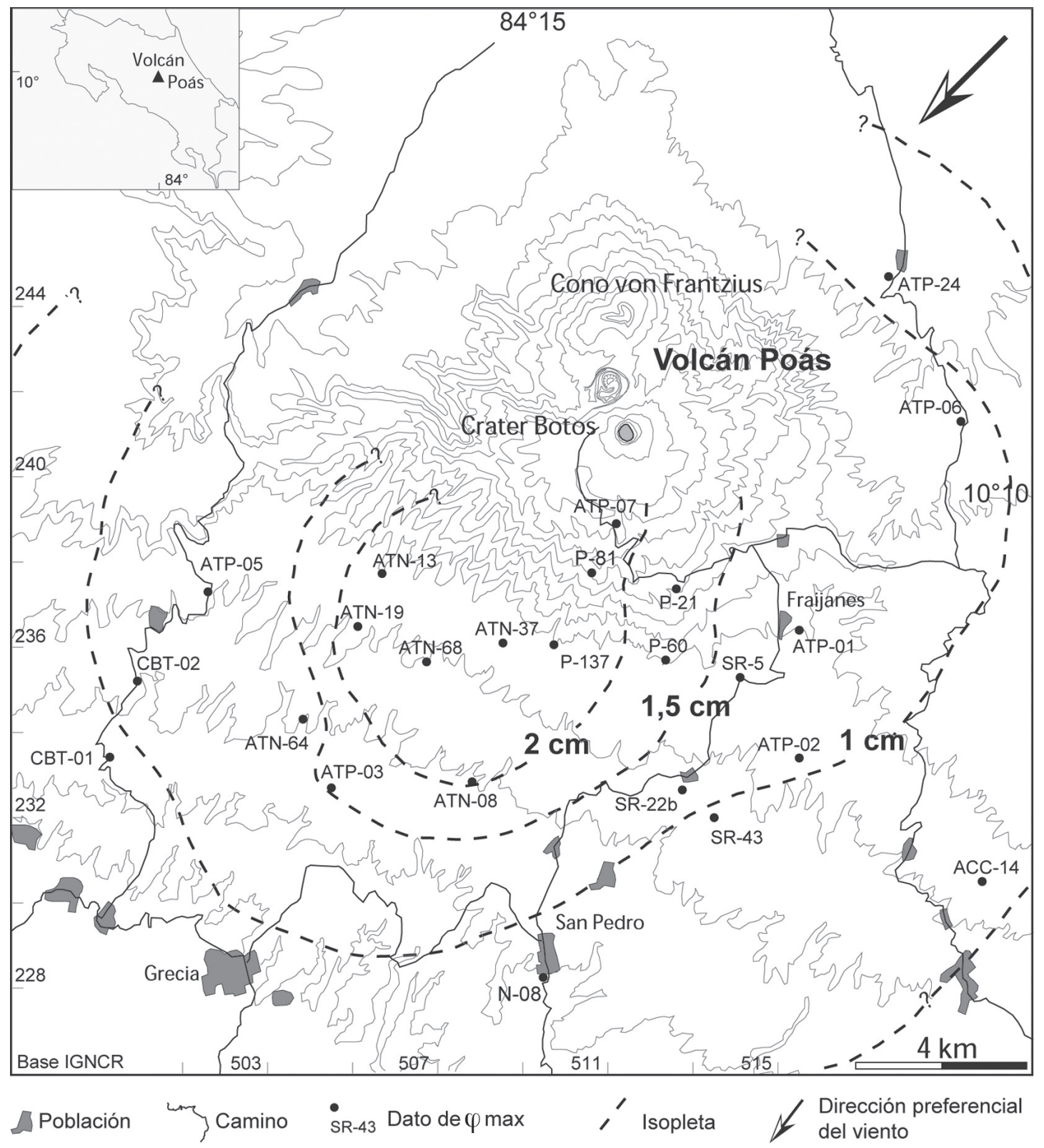

Fig. 5. Mapa de isopletas ( $\varphi$ máx.) de la capa superior de al Toba de Lapilli Poás, mediciones ATP de Prosser (1983); N-08, ACC y ATN (N. Montes, com. pers., 2006) y SR, P y CBT de los autores. 


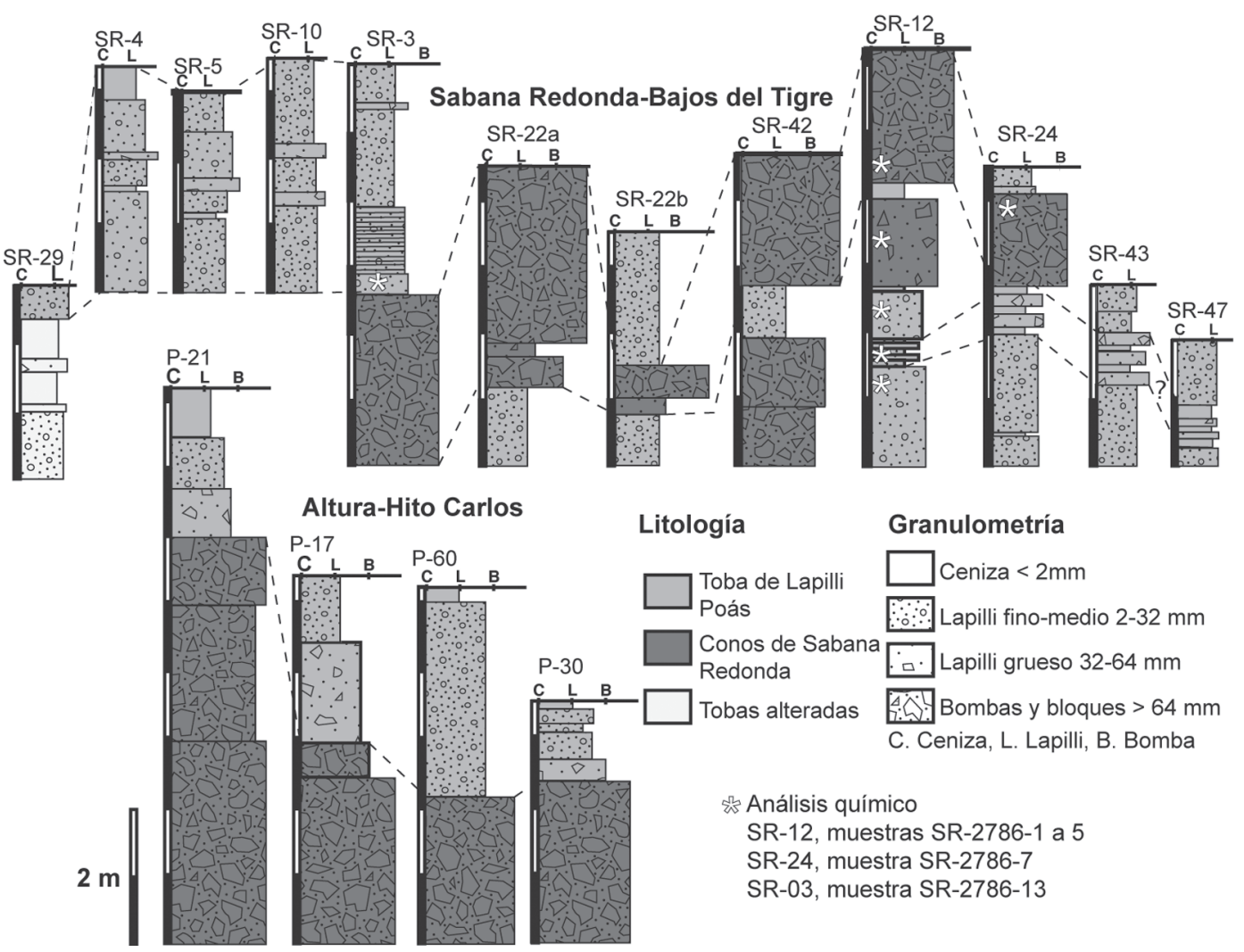

Fig. 6. Tefroestratigrafía de la Toba de Lapilli Poás y los conos piroclásticos de Sabana Redonda.

\section{Falla Carbonera}

Definida por Alvarado et al. (1988), se ubica en el flanco oeste del volcán Poás. Presenta una dirección nor-noroeste y con pendientes mayores a $30^{\circ}$. El escarpe de esta falla se extiende $2 \mathrm{~km}$ en el sector noroeste de la zona de estudio con un rumbo noroeste-sureste (Fig. 1). Las evidencias fotogeológicas sugieren un movimiento relativo dextral con base en el desplazamiento de las divisorias de agua en ese sentido.

\section{GEOQUÍMICA}

\section{Resultados}

Desde un punto de vista geoquímico, las lavas del volcán Poás varían de basaltos a dacitas, siendo en su mayoría andesitas basálticas y andesitas. Las muestras de Sabana Redonda (los conos piroclásticos y las andesitas Poasito) y Paleo-Poás son relativamente más altas en álcalis y por lo tanto presentan una tendencia traquítica (Prosser \& Carr, 1987; Patino et al., 2000; Carr, 2002).

En los alrededores de los conos piroclásticos Sabana Redonda, se encuentran intercalaciones de depósitos de la Toba Lapilli Poás con depósitos de bloques y bombas proximales y depósitos de lapilli distales de los conos piroclásticos. Los criterios macroscópicos y microscópicos no son suficientes para diferenciar entre la Toba de Lapilli Poás y de los depósitos distales de los conos.

Las muestras SR-2786-1 a SR-2786-5 (Figs. 6 y 10) representan un muestreo estratigráfico de la sección medida SR-12. Los resultados de algunos de los elementos mayores analizados se muestran el la figura 10. Los depósitos de los 


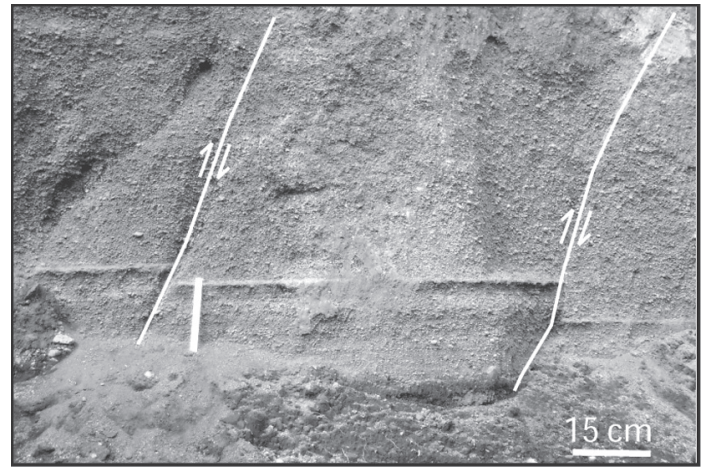

Fig. 7. Parte de la sección inferior de la Toba de Lapilli Poás $(513,5 / 232,8)$.

conos piroclásticos Sabana Redonda, presentan mayores contenidos de $\mathrm{TiO}_{2}(>1 \%)$ y de $\mathrm{Fe}_{2} \mathrm{O}_{3}$ que inclusive superan el $11 \%$, así como las menores concentraciones de $\mathrm{CaO}(<6,5 \%)$. Las concentraciones de $\mathrm{Al}_{2} \mathrm{O}_{3}$ son variables en ambas unidades.

Además de las muestras anteriores se analizó una muestra de los depósitos distales de los Conos de Sabana Redonda en la sección medida SR-24 (muestra SR-2786-13, Fig. 6) y la Toba de Lapilli Poás en la sección SR-3 (muestra SR2786-7, Fig. 6) con concentraciones de $\mathrm{TiO}_{2}$ de $1,1 \%$ y $0,91 \%$ respectivamente, lo cual coincide con las concentraciones de la sección SR-12 y los valores reportados por Prosser \& Carr (1987) para estas unidades.

\section{Componentes magmáticos del volcán Poás}

Los diagramas de variación (Fig. 11) evidencian dos poblaciones bien definidos en el volcán Poás, las cuales representan dos tipos de magma diferentes: uno enriquecido en elementos de alto equipotencial (HFSE, $\mathrm{TiO}_{2}, \mathrm{Zr}, \mathrm{Nb}$ ) y otros elementos incompatibles como $\mathrm{P}_{2} \mathrm{O}_{5}$ y $\mathrm{K}_{2} \mathrm{O}$ y otro empobrecido en los elementos mencionados. Ambos comparten concentraciones similares de $\mathrm{SiO}_{2}, \mathrm{Al}_{2} \mathrm{O}_{3}, \mathrm{MgO}$ y $\mathrm{Ba}$, lo que sugiere que estas rocas presentan patrones evolutivos de diferenciación similares (Fig. 11).

El componente enriquecido o componente Sabana Redonda $\left(\mathrm{TiO}_{2}>1 \%\right)$, está representado por las lavas y depósitos piroclásticos de los conos Sabana Redonda y además, se encuentra presente en las lavas Paleo-Poás, la Toba de Lapilli Poás y algunas lavas del cráter Botos altas en $\mathrm{TiO}_{2}$.

El componente empobrecido o componente von Frantzius $\left(\mathrm{TiO}_{2}<0,8 \%\right)$, se encuentra presente en las lavas de la zona del cráter principal, von Frantzius, la laguna de Hule y lavas del cráter Botos.

El componente Sabana Redonda (Fig. 12), se encuentra enriquecido en elementos de tierras raras livianas en factores de aproximadamente $20 \%$ con respecto al componente von Frantzius. Sin embargo, esta diferencia no es tan evidente en los elementos de tierras raras pesadas (aproximadamente 5\%). Estos factores y las concentraciones similares de Ba (Fig. 11) en ambos componentes sugiere que se originaron a partir de una misma fuente, por medio de diferentes grados de fusión parcial (Haskin, 1984), siendo mayor el grado de fusión parcial para el componente von Frantzius.

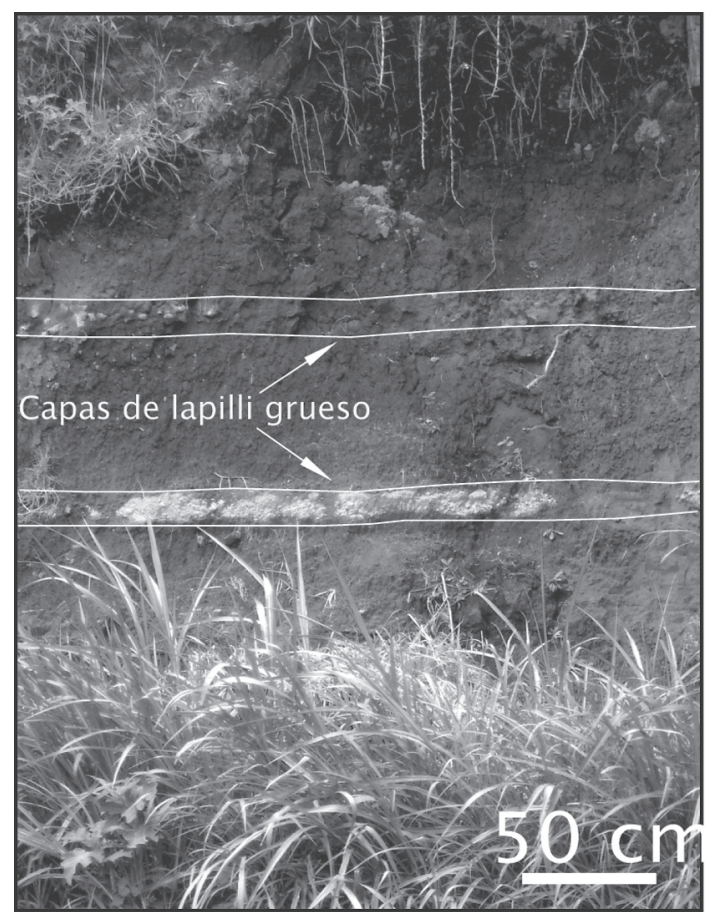

Fig. 8. Parte de la sección superior de la Toba de Lapilli Poás $(514,07 / 235,09)$. 




Fig. 9. Tobas alteradas con intercalaciones de eventos félsicos (capas color gris claro) que subyacen la Toba de Lapilli Poás $(512,3 / 232,114)$.

Las razones $\mathrm{Zr} / \mathrm{Nb}(10-16), \mathrm{Ba} / \mathrm{La}(15-35)$ son las más bajas para el componente Sabana Redonda y las más altas para el componente von Frantzius, lo que sugiere que estos magmas se originaron a partir de diferentes grados de fusión parcial de una fuente mantélica OIB (las razones $\mathrm{Zr} / \mathrm{Nb}$ y Ba/La son 6 y 10 respectivamente en un OIB sensu Sun \& MacDonough, 1989) modificada por procesos de subducción.

La mayoría de las lavas del Poás representan híbridos con composiciones químicas entre los extremos de los componentes Sabana Redonda y von Frantzius. Con el propósito de cuantificar aproximadamente los porcentajes de mezcla que intervienen en la génesis de estas lavas, se seleccionaron las muestras con un rango de $\mathrm{SiO}_{2}$ de (50-60\%) y $\mathrm{TiO}_{2}(0,7-1,1 \%)$ con el objetivo de evitar los efectos extremos de cristalización fraccionada. Con base en esto se construyeron dos modelos, el primero con los porcentajes de $\mathrm{TiO}_{2}$ $\mathrm{SiO}_{2}$ (Fig. 13a) y el segundo con los elementos de tierras raras (Fig. 13b). Se utilizaron las ecuaciones de mezcla descritas por Albarède (1995).
Con base en estos modelos (Fig. 13) se sugiere que los magmas con $\mathrm{TiO}_{2}>1 \%$, requieren al menos $80 \%$ del componente Sabana Redonda. Las muestras con un rango de $\mathrm{TiO}_{2}$ de $0,8-1 \%$, requirieron de una mezcla de al menos $20 \%$ del componente Sabana Redonda y $80 \%$ del componente von Frantzius. Los magmas con $\mathrm{TiO}_{2}$ $<0,8 \%$, necesitaron un porcentaje mayor a $80 \%$ del componente von Frantzius. El modelo de mezcla hecho a partir de los elementos de tierras raras (Fig. 13b) con un $40 \%$ del componente Sabana Redonda y $60 \%$ del componente von Frantzius coincide con la composición química de la Toba de Lapilli Poás.

\section{DISCUSIÓN Y CONCLUSIONES}

Las características petrográficas y geoquímicas de las andesitas Poasito y los conos piroclásticos Sabana Redonda indican que se formaron a partir de un mismo magma con variaciones en las características reológicas (viscosidad, volátiles, etc). Estas unidades cogenéticas se originaron por medio de un vulcanismo fisural caracterizado por las andesitas Poasito en las etapas efusivas y los conos piroclásticos en las etapas explosivas. En ambos casos el magma ascendió rápidamente (características afíricas) y posteriormente entró en contacto con condiciones atmosféricas a alta temperatura (oxidación espontánea en los conos piroclásticos y texturas fluidales en las coladas) (Prosser \& Carr, 1987).

Las secciones medidas (SR-12, SR-42, Fig. 6) muestran que los depósitos de los conos piroclásticos ocasionalmente se encuentran separados por tobas de lapilli o ceniza. Las diferentes coladas de las andesitas Poasito, genéticamente relacionadas con los conos piroclásticos, también se encuentran separadas por tobas. Estas evidencias sugieren que los conos piroclásticos no fueron contemporáneos, sino que más bien corresponden con diferentes pulsos de la actividad fisural. Dataciones radiométricas $\mathrm{Ar} / \mathrm{Ar}$ en proceso ayudarán a comprobar estas evidencias.

Según la datación radiométrica C-14 reportada por Prosser \& Carr (1987), la edad de la Toba de Lapilli Poás es mayor a los 40 ka, sin embargo los depósitos de los conos piroclásticos 

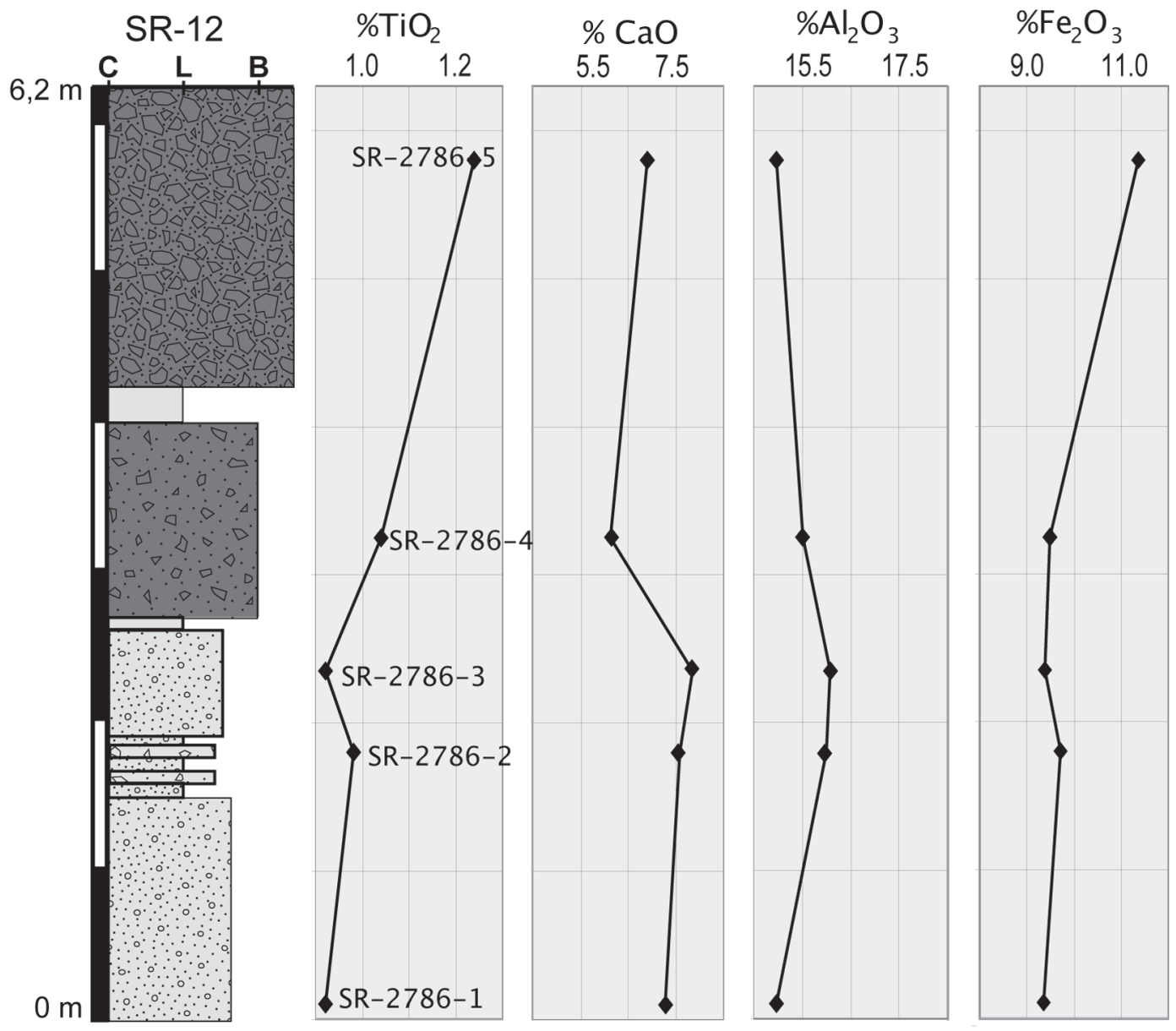

Fig. 10. Resultados de las variaciones de elementos mayores entre la toba de lapilli Poás y los conos piroclásticos de Sabana Redonda.

que se encuentran intercalados entre la sección superior e inferior de de esta toba (Fig. 6), tiene una edad de 33 ka según una datación C-14 (G.E. Alvarado, com. pers., 2005). Esto implica que la sección datada por Prosser \& Carr (1987) corresponda con la sección inferior de la toba de lapilli Poás que subyace los conos piroclásticos Sabana Redonda.

La composición química de la Toba de Lapilli Poás, es similar a las muestras del cráter Botos, ambas altas en $\mathrm{TiO}_{2}$ (Fig. 11). Las isopletas de $\varphi$ máx. de los componentes juveniles de la sección superior de esta toba (Fig. 5), sugieren que la fuente corresponde con el sector cuspidal del volcán Poás. Por lo tanto es posible que esta unidad este relacio- nada al cráter Botos, ya que la composición geoquímica de las muestras del cráter principal es bastante diferente a las muestras de esta toba (Fig. 11).

Los bloques encontrados dentro de los depósitos de los conos piroclásticos representan porciones del basamento local (andesitas basálticas Achiote y andesitas Poasito) y bloques de los conductos profundos de los conos piroclásticos (Paleo-Poás).

Con base en los análisis químicos disponibles (Prosser \& Carr, 1987, Patino et al., 2000; Carr, 2002), se definieron dos componentes magmáticos para el volcán Poás (Figs. 11 y 12), que son: 1) El componente Sabana Redonda, enriquecido en HFSE y otros elementos incompatibles 


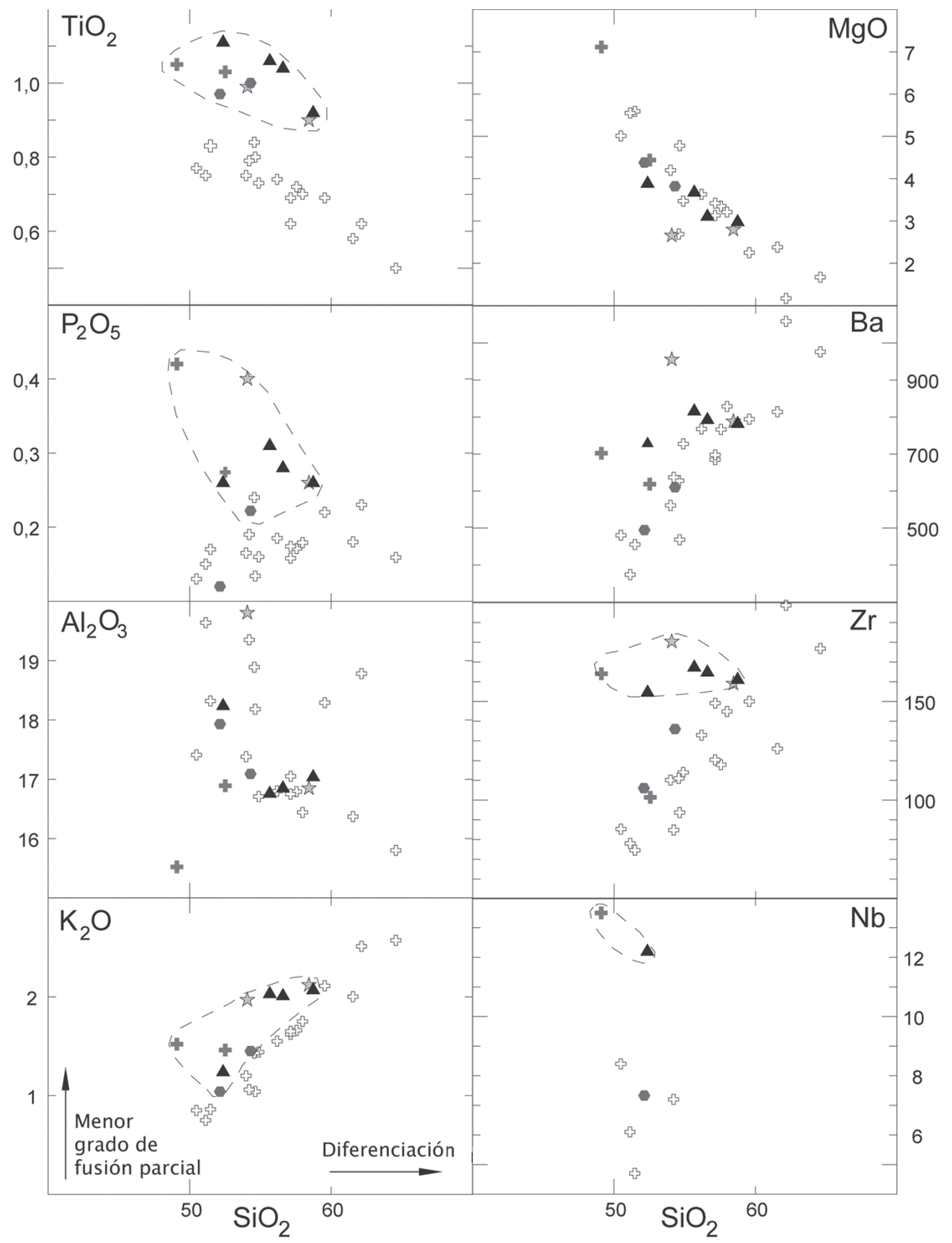

\section{- Sabana Redonda \\ Botos alto en $\mathrm{Ti}$ \\ Toba de Lapilli Poás is Paleo-Poás \\ ¡ Poás bajo en Ti}

Fig. 11. Diagramas de variación y componentes magmáticos del Volcán Poás. Las muestras entre líneas discontinuas representa el componente Sabana Redonda. Los datos geoquímicos son de:Prosser \& Carr 1987, Patino et al., 2000 y Carr, 2002). Los elementos mayores (óxidos en \%) y las trazas en ppm. 


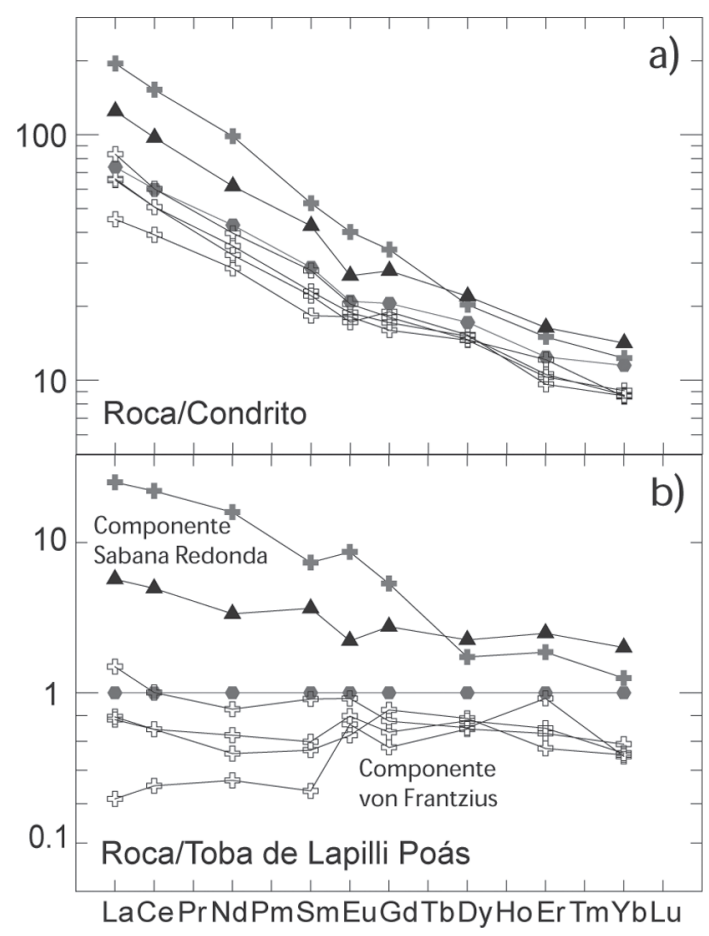

Fig. 12. Patrones de elementos de tierras raras del volcán Poás. a) Normalización a condritos según Sun \& MacDonough (1989); b) Normalización de los datos con la Toba de Lapilli Poás con el propósito de mostrar los dos componentes del volcán Poás. Los datos geoquímicos son de Patino et al., 2000. Los símbolos son los mismos de la Fig. 11.

$\left.\left(\mathrm{K}_{2} \mathrm{O}, \mathrm{P}_{2} \mathrm{O}_{5}\right) .2\right) \mathrm{El}$ componente von Frantzius, que está relativamente empobrecido en HFSE y en otros elementos incompatibles mencionados, originado a partir de un mayor grado de fusión parcial que el componente Sabana Redonda. La mezcla de estos dos componentes explica la composición química de la mayoría de las lavas del Poás analizadas hasta el momento. Ambos componentes comparten una misma fuente tipo OIB, la cual fue modificada por los procesos de subducción. El mecanismo de fusión para el componente Sabana Redonda se interpreta como producto de un proceso de decomprensión adiabática y para el componente von Frantzius por una fusión por presencia de fluidos provenientes de la zona de subducción (Cameron et al., 2002). Los componentes magmáticos del Poás, son análogos con los resultados encontrados en el volcán Irazú por Alvarado et al. (2006).

El proceso de decomprensión adiabática puede estar relacionado con la extensión generada dentro de la fractura volcanotectónica del
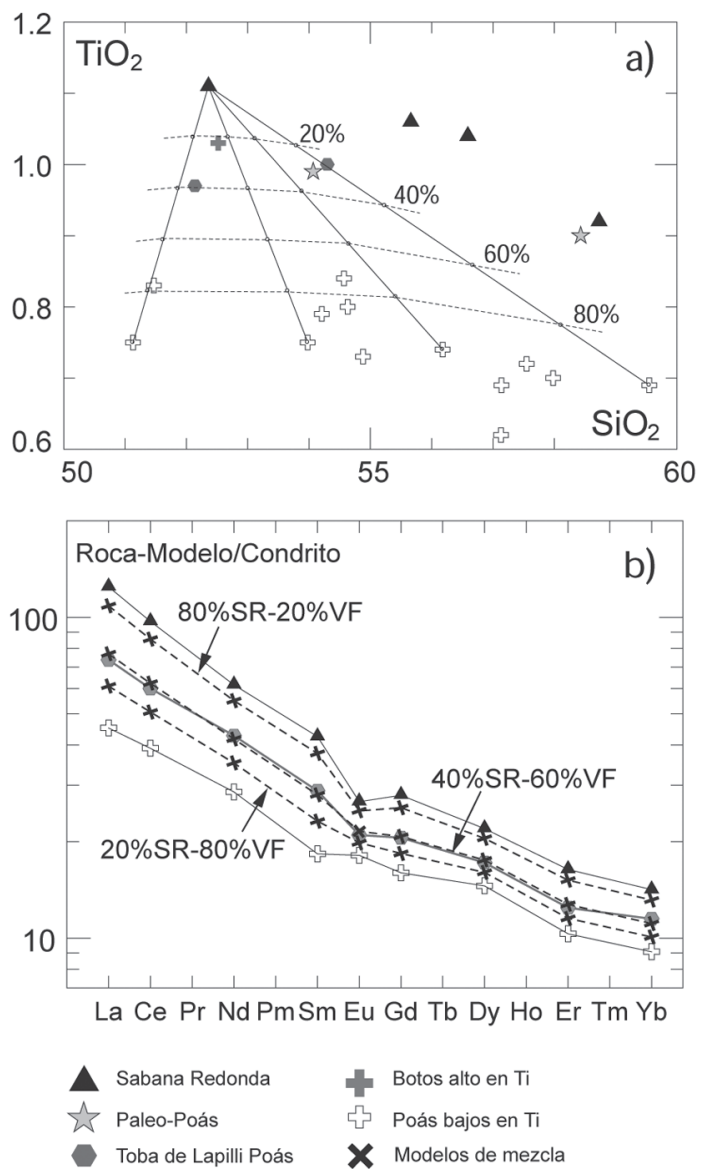

Fig. 13. Modelos de mezcla entre los componentes del volcán Poás. A) Modelo lineal con base en $\mathrm{TiO}_{2}-\mathrm{SiO}_{2}$; B) Modelo de mezcla de los elementos de tierras raras normalizados a condritos (Sun \& MacDonough 1989). Las muestras de los extremos de los dos componentes son las mismas en el modelo lineal más a la izquierda en A y los patrones de tierras raras en B. Los otros extremos del componente von Frantzius en el modelo $\mathrm{SiO}_{2}-\mathrm{TiO}_{2}$ representan muestras más fraccionadas, no obstante los resultados coinciden a pesar de fraccionamiento. SR, componente Sabana Redonda; VF, componente von Frantzius. 
Poás, producto de un esfuerzo horizontal máximo regional de dirección norte-sur para la parte central de Costa Rica (Montero, 1994).

\section{AGRADECIMIENTOS}

Se agradece a CEMEX de Costa Rica por realizar los análisis químicos en sus laboratorios. A los profesores Michael Carr y Lina Patino por las discusiones y comentarios de la interpretación geoquímica. A los profesores Siegfried Kussmaul, Gerardo Soto y Luis Guillermo Obando, por las discusiones durante las primeras etapas del trabajo de campo. Al Profesor Guillermo Alvarado por suministrar la datación C-14 de los conos piroclásticos Sabana Redonda y por la motivación de escribir este artículo. A Natalia Montes por compartir sus datos de $\varphi$ max. Por último se agradece a todos los que en algún momento cooperaron en el trabajo de campo durante la Campaña Geológica del 2004 y las posteriores etapas de esta investigación.

\section{REFERENCIAS}

ALBARÈDE, F., 1995: Introduction to geochemical modeling.- 543 págs. Cambridge Univer. Press, New York.

ALVARADO, G. E., 2000: Los volcanes de Costa Rica: geología, historia y riqueza natural.269 págs. UNED, San José, Costa Rica.

ALVARADO, G.E., CARR, M.J., TURRIN, D. D., SWICHER III, C.C., SCHMINCKE, H.-U. \& HUDNUT, K.W., 2006: Recent volcanic history of Irazú volcano, Costa Rica: Alternation and mixing of two magma batches, and pervasive mixing.En: ROSE, W.I., BLUTH, G.J.S., CARR, M.J., EWERT, J., PATINO, L.C., AND VALLANCE, J. (eds.): Volcanic hazards in Central America, Geol. Soc. Amer. Spec. Pap. 412: 259-276.

ALVARADO, G. E., MORALES, L. D., MONTERO, W., CLIMENT, A. \& ROJAS,
W., 1988: Aspectos sismológicos y morfotectónicos en el extremo occidental de la cordillera volcánica Central de Costa Rica.Rev. Geol. América Central, 9: 75-98.

BARQUERO, J. \& MALAVASSI, R. E., 1982: Excursión al volcán Poás.- Bol. Volcan. 14:120-131, UNA, Heredia.

CAMERON, B. I., WALTER, J. A., CARR, M.J., PATINO, L.C., MATIAS, O. \& FEIGENSON, M.D., 2002: Flux versus decompression melting at stratovolcanonoes in southeastern Guatemala.- J. Volcanol. Geotherm. Res. 119: 21-50.

CARR, M. J. 2002: CAGeochem database.http://www.rci.rutgers.edu/ carr/, consulta setiembre 2006.

DENYER, P., MONTERO, W. \& ALVARADO, G., 2003: Atlas tectónico de Costa Rica.81 págs. Ed. Univ. Costa Rica, San José.

DeMETS C., 2001: A new estimate for presentday Cocos-Caribbean plate motion: Implication for slip along the Central American volcanic arc.- Geophys. Res. Let. 28(21): 4043-4047.

FISHER, R.V. \& SCHMINCKE, H.U., 1984: Pyroclastic rocks.- 472 págs. Springer, Berlin.

HASKIN, L.A., 1984: Petrogenetic modellinguse of rare earth elements.- En: HENDERSON, P.: Rare earth elements geochemistry. - 510 págs. Elsevier, Amsterdam.

PROSSER, J.T., 1983: The geology of Poás volcano, Costa Rica.- 165 pags. Darmouth Collegue, New Hampshire [Tesis MSc.].

PROSSER, J.T. \& CARR, M.J., 1987: Poás volcano, Costa Rica: Geology of the summit region and spatial and temporal variations among the most recent lavas.- J. Volcanol. Geotherm. Res. 33: 131-146. 
SUN, S-S. \& McDONOUGH, W.F., 1989: Chemical and isotopic systematics of oceanic basalts: Implications for mantle copositions and processes. - En: SAUNDERS, A.D. \& NORRY, M.J. (eds.): Magmatism in the Ocean Basins.- Geol. Soc. Am. Spec. Pap. 42: 313-345.
TOURNON, J., 1984: Magmatismes du mesozoique a 1 'actuel en Amerique Centrale: L 'exemple de Costa Rica, des ophiolites aux andesites.- 335 págs., Mem. Sc. Terre Univ. Curie, Paris [Tesis $\mathrm{PhD}$ ]. 